\title{
Cold cathode rf guns based study on field emission
}

\author{
Xiangkun Li, ${ }^{1,2, *}$ Ming Li, ${ }^{2}$ Lijun Dan, ${ }^{2}$ Yu Liu, ${ }^{2}$ and Chuanxiang Tang ${ }^{1}$ \\ ${ }^{1}$ Department of Engineering Physics, Tsinghua University, Beijing 100086, China \\ ${ }^{2}$ Institute of Applied Electronics, China Academy of Engineering Physics, Mianyang 621900, China
}

(Received 19 August 2013; published 5 December 2013)

\begin{abstract}
Recently cold cathodes based on field emission have drawn attention and been considered to drive accelerators and free electron lasers, due to the progress in field emitter arrays and planar emitters like diamond films. In this paper, we reviewed the characteristics of field emission in rf fields. Simulations of S-band rf guns consisting of a cathode cell and a full cell were done. We showed that a shorter cathode cell with a length of $0.25-0.3$ of $\lambda / 2$ is in favor of obtaining both low emittance and low energy spread bunches when the amplitude of electric field on the cathode surface ranges from 60 to $80 \mathrm{MV} / \mathrm{m}$. A single cell test cavity has been installed to study field emission of diamond films and the measured beam current showed a good agreement with theoretical calculations.
\end{abstract}

DOI: 10.1103/PhysRevSTAB.16.123401

PACS numbers: $79.70 .+q, 29.25 . B x$

\section{INTRODUCTION}

Radio-frequency (rf) guns have been considered the most promised electron guns in many applications for their high accelerating gradient, which is essentially important to suppress the space charge induced growth of transverse emittance. In recent decades, rf guns based on thermionic cathodes and photocathodes have been successfully developed [1]. Although both types of guns have desirable characteristics, there are also limitations. For thermionic rf guns, the average beam current is high due to the continuous emission of electrons in every rf cycle. But the ungated emission degrades the beam quality, and the back-bombardment from electrons emitted late in the rf cycle threatens the stable operation and lifetime of the cathode. Photocathode rf guns use lasers to gate the emission and can produce very bright electron bunches with high peak current. But the average current is restricted by the low repetition rate of drive lasers.

The field emission cathode or cold cathode uses the strong electrical field to directly pull electrons from the cathode surface. Like thermionic cathodes, cold cathodes can emit electrons in every rf cycle to obtain high average current. In addition, field emission is concentrated around the peak of the electrical field within a small portion of the rf cycle $\left( \pm 30^{\circ}\right)$ so the beam quality can be better controlled.

The idea of cold cathode rf guns is not new and has been tried from time to time [1,2]. The biggest difficulty of such a gun is the absence of suitable field emitters. In recent years, new fabrication technologies and ideas have

\footnotetext{
*lixiangkun05@sina.com

Published by the American Physical Society under the terms of the Creative Commons Attribution 3.0 License. Further distribution of this work must maintain attribution to the author(s) and the published article's title, journal citation, and DOI.
}

opened ways for such uses. For example, the diamond field emitter arrays can produce a highest current density of $\sim 100 \mathrm{~A} / \mathrm{cm}^{2}$ [3]. Planar emitters like diamond films (DF) have also shown the potential of high emission current [4]. In the Institute of Applied Electronics of China Academy of Engineering physics (CAEP/IAE), attempts to build a cold cathode rf gun are being done. The gun is going to be a part of a terahertz free electron laser facility.

In this paper, preparations for the construction of a cold cathode rf gun are presented. In the second section, the basic characteristics of such a combination is described. Beginning with the Fowler-Nordheim equation, we show the numerical results of field emission in an rf cycle and during the building of electromagnetic field in the gun. The temporal distribution of electrons with respect to the $\mathrm{rf}$ phase is approximately Gaussian, with an rms width of about $15^{\circ}$ for a very large range of operation conditions. The start point of emission during the field building is much later than the feeding of the rf power by roughly twice the time constant of the gun, which has been observed in our experiments.

In the third section, simulations of a S-band $\mathrm{rf}$ gun consisting of a cathode cell and a full cell for cold cathodes are discussed. Since the injection phase is $\pi / 2$, the cathode cell length should be shortened to obtain both efficient acceleration and good transverse emittance. We show that a length of $0.25-3$ of $\lambda / 2$ for the cathode cell is preferred with the amplitude of electrical field from 60 to $80 \mathrm{MV} / \mathrm{m}$. The transverse emittance and energy spread of the extracted beams could be small. In CAEP/IAE, a single cell test cavity has been constructed based on which we have measured the emission of a few diamond films. The highest beam current we have observed was $9 \mathrm{~mA}$ (or $3.15 \mathrm{pC}$ in charge). Details of the experiments are contained in the fourth section. Finally, a summary of this paper is given. 


\section{CHARACTERISTICS OF COLD CATHODE RF GUNS}

The field emission from an infinite smooth metallic surface can be described by the famous Fowler-Nordheim equation [5],

$$
J(E)=a \frac{E^{2}}{\Phi} \exp \left(\frac{b}{\sqrt{\Phi}}\right) \exp \left(-c \frac{\Phi^{\frac{3}{2}}}{E}\right),
$$

where $J$ is the emission current density, $E$ is the applied electric field strength, $\Phi$ is the work function, and $a, b, c$ are constants. To obtain a useful amount of charge (for free electron lasers, $\sim 100 \mathrm{pC}$ ) it requires a very strong electrical field that is too high to achieve. Figure 1 shows the dependence of $J$ on $E$ for a planar emitter made of copper. The inset of it shows the exponential relationship. Several $\mathrm{GV} / \mathrm{m}$ is necessary to obtain the same emission intensity $\left(\sim 10 \mathrm{~A} / \mathrm{cm}^{2}\right)$ as thermionic cathodes. Therefore, field emitter arrays are favored. As the emitting spot becomes smaller, the necessary field applied on the tips should be higher.

\section{A. Field emission in an rf cycle}

In the rf gun, the electric field on the cathode surface varies as $E_{0} \cos (\omega t)$, where $E_{0}$ is the amplitude of electrical field, $t$ is time, and $\omega$ is the angular frequency. Insertion of $E_{0} \cos (\omega t)$ into Eq. (1) and a few algebraic operations will give [6]

$$
J \sim a^{\prime} \exp \left(-c \frac{\Phi^{\frac{3}{2}}}{E_{0}} \frac{(\omega t)^{2}}{2}\right) .
$$

Let

$$
\frac{1}{2 \sigma^{2}}=\frac{c}{2} \frac{\Phi^{\frac{3}{2}}}{E_{0}}
$$

We find that $J \sim a^{\prime} \exp \left[-(\omega t)^{2} / 2 \sigma^{2}\right]$, which means the temporal distribution of field emission is Gaussian with respect to the rf phase or time. Again taking copper as an

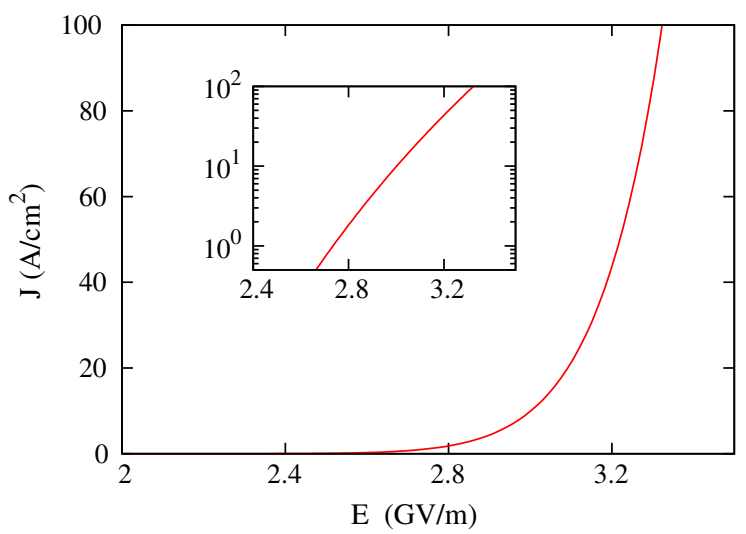

FIG. 1. Field emission from a planar copper surface. The work function of copper used here is $4.65 \mathrm{eV}$. The inset shows the relation between $\ln J$ and $E$.

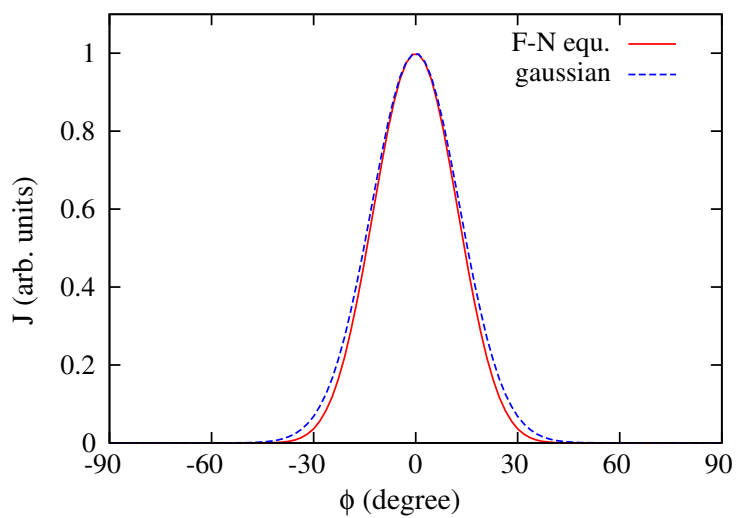

FIG. 2. Temporal distribution of field emission with the current density normalized for comparison. A material of copper is supposed and the peak emission density is $100 \mathrm{~A} / \mathrm{cm}^{2}$. The resulted rms width is $12.9^{\circ}$.

example, for a peak emission density of $100 \mathrm{~A} / \mathrm{cm}^{2}$ (when the electrical field is at its maximum), $\sigma=12.9^{\circ}$. We plotted the theoretical distribution by Eq. (1) and Gaussian distribution by Eq. (3) in Fig. 2. They agree well with each other.

It is meaningful to know to what extent the rms width $\sigma$ would go when the work function $\Phi$ and the amplitude of electrical field $E_{0}$ are changed. We calculated $\sigma$ for different combinations of $\Phi$ and $E_{0}$ with a constant peak emission density $J_{0}$, as shown in Fig. 3. It turns out that $\sigma$ is hardly changed for given $J_{0}$. When increasing $J_{0}, \sigma$ becomes larger but the difference is small.

\section{B. Field emission during a macropulse}

A rf gun is often operated in the macropulse mode. Every macropulse includes thousands of rf cycles. At the beginning of the macropulse, the amplitude of electrical field is growing with the energy stored in the cavity. Without considering beam loading, the stored energy in the gun cavity is given by [7]

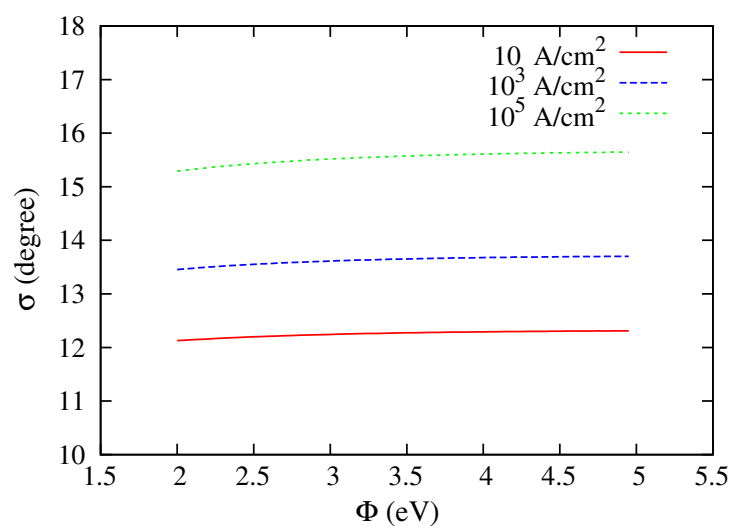

FIG. 3. The rms width of field emission in an rf cycle. For variant emission current densities and work functions, the width only changes a little. 


$$
U(t)=\frac{Q_{0}}{\omega} \frac{4 \beta}{(1+\beta)^{2}}\left(1-e^{-t / \tau}\right)^{2} P_{+}=U_{s}\left(1-e^{-t / \tau}\right)^{2},
$$

where $Q_{0}$ is the unloaded quality factor, $\beta$ is the waveguide-to-cavity coupling strength, $\tau=2 Q_{0} / \omega(1+\beta)$ is the time constant, $P_{+}$is the forward power, and $U_{s}$ is the stored energy at steady state.

The transient amplitude of the electric field on the cathode surface is related to the stored energy by $U=$ $\alpha E^{2}$ and at steady state it becomes $U_{s}=\alpha E_{s}^{2}$. Replacing $U$ and $U_{s}$ in Eq. (4) by $E$ and $E_{s}$, respectively, gives

$$
E(t)=E_{s}\left(1-e^{-t / \tau}\right) .
$$

For the thermionic cathode, the emission occurs in every rf cycle and the bunch charge is mainly determined by the temperature of the cathode. The electrical field may enhance the emission because the Schottky effect will lower the surface barrier but its effect is limited. The beam current will rise rapidly and reach its peak immediately after the feeding of rf power. The back-bombarding electrons can cause a significant rise in the temperature, resulting in an increase of the beam current [8] during the macropulse. For the photocathode, the emission is excited by a laser pulse and the bunch charge depends on the quantum efficiency and the laser power. The timing and power of laser pulse in every macropulse is the same so there is no difference in bunch charge. Unlike thermionic emission and photoemission, field emission is very sensitive on the electrical field. If the amplitude of electrical field is small, the emission in that cycle will be too low to be observed. By integrating and averaging Eq. (2) within one rf cycle we get the average beam current, which we can measure by a Faraday cup or a current transformer,

$$
I=S \times a \frac{E^{2.5}}{\Phi^{1.75}} \exp \left(\frac{b}{\Phi^{0.5}}\right) \exp \left(-c \frac{\Phi^{1.5}}{E}\right),
$$

where $S$ is the area of the cathode and $E$ is the transient amplitude of electrical field. From Eq. (6) we get

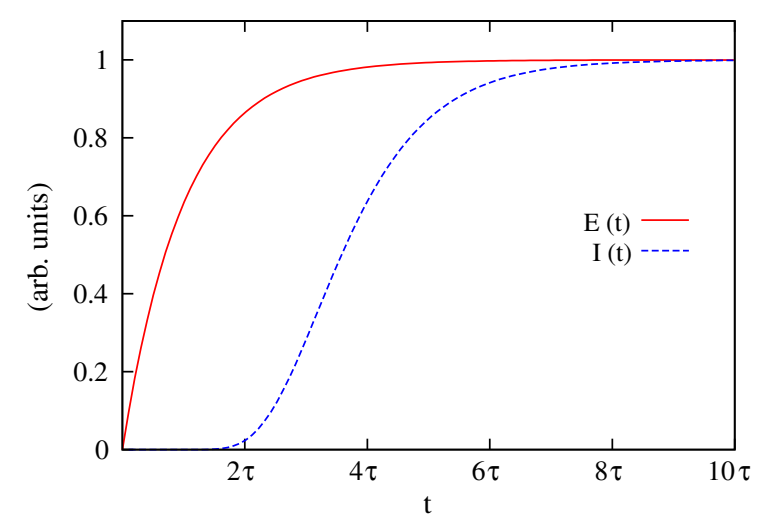

FIG. 4. Amplitude of electrical field and average beam current vs time during a macropulse. Here $\tau=0.33 \mu \mathrm{s}$. $\ln \left(I / E^{2.5}\right) \propto-c \Phi^{1.5} / E$. The linear dependence of $\ln \left(\bar{I} / E^{2.5}\right)$ on $1 / E$ can be used as proof of field emission.

By inserting Eq. (5) into Eq. (6) we have

$$
I(t)=I_{s}\left(1-e^{-t / \tau}\right)^{2.5} e^{-\frac{b}{E(t) 1-e^{-t / \tau}}} .
$$

Here $I_{s}$ is the average current at steady state.

Figure 4 shows the trend of the amplitude of the electrical field and the average current with time during a macropulse. It takes nearly $2 \tau$ to build a field strong enough for observable emission. Compared with thermionic cathode, a relatively longer macropulse is needed to get a flat beam current.

\section{SIMULATIONS OF A TWO CELLS RF GUN FOR FIELD EMISSION}

\section{A. Considerations}

The basic structure of the rf gun used here consists of a cathode cell and a full cell (see Fig. 5) with the cold cathode inserted into the rear wall of the cathode cell. As said above, field emission in an $\mathrm{rf}$ cycle is concentrated around the phase of $\pi / 2$ with an rms width of about $15^{\circ}$. The advantage of an injection phase of $\pi / 2$ is that the space charge could be suppressed immediately after emission. But it accompanies other disadvantages. Since the initial velocity of electrons is nearly zero, it takes more than half an rf period to travel from the cathode to the full cell. Therefore, the cathode cell length should be adjusted such that the electron beam transit time corresponds to a good arrival phase at the entrance to the full cell. By doing so, the rf effect on transverse emittance can be suppressed at the same time. Recalling Kim's theory [9], the transverse emittance is minimized when the phase $\phi=\omega t-k z+\phi_{0}$ near the gun exit is $\pi / 2$. Here $\lambda$ is the wavelength, $k=2 \pi / \lambda$. Although it is derived for a $n+\frac{1}{2}$ cells gun, Kim's conclusion is valid for guns with different cathode cell lengths. A rule of thumb is that a longer cathode cell requires an earlier injection phase and vice versa. For a photocathode gun, the cathode cell length is usually increased to shift $\phi_{0}$ to be

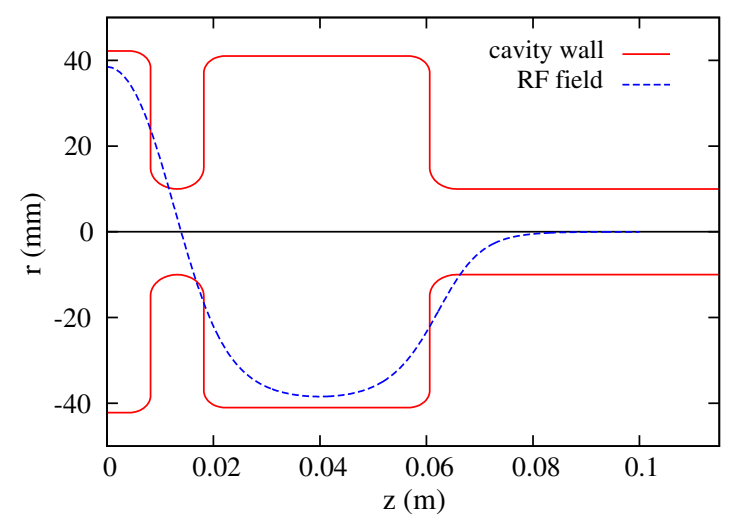

FIG. 5. Cavity wall and longitudinal field distribution of a 1.25 cells gun. 


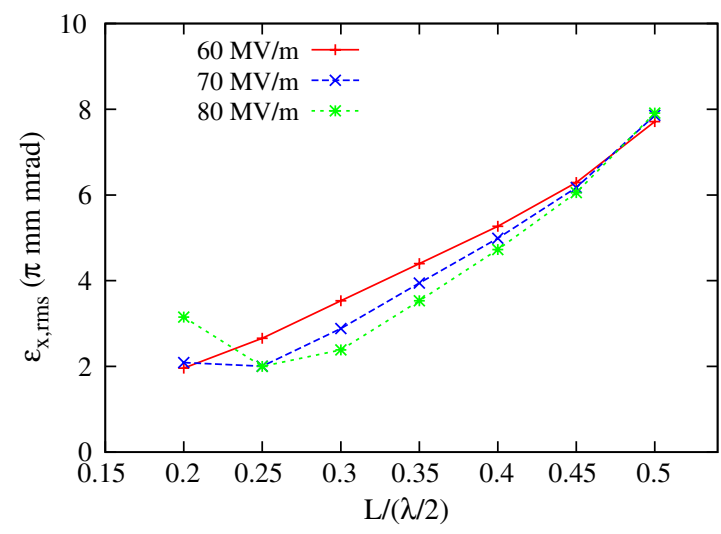

FIG. 6. Transverse emittance vs cathode cell length.

closer toward $0^{\circ}$. For a cold cathode gun, the fixed injection phase requires a shorter cathode cell.

The cathode cell length also depends on the accelerating gradient. A higher gradient means electrons can reach the full cell earlier so a relatively long cathode cell can be employed. The power of our klystron suggests a gradient from 60 to $80 \mathrm{MV} / \mathrm{m}$. The necessary beam current to drive the terahertz free electron laser is about $200 \mathrm{~mA}$ (or a bunch charge of $70 \mathrm{pC}$ ). The current is peaked at $\pi / 2$ with an rms width of $15^{\circ}$ and emitted from a cathode with a radius of $1 \mathrm{~mm}$. By varying cathode cell length and gradient, the dynamics in the gun was tracked by ASTRA [10]. The space charge was turned off so only the rf effect was evaluated.

Since the emission occurs within a large portion of the rf period, some electrons may fall into decelerating phase inside the gun, especially those emitted late. Like that in a thermionic rf gun, the energy spread of the extracted electron bunch is large. The beam tail that corresponds to the low energy part of the beam will expand in the following transportation and degrade the beam quality. A commonly used solution is getting rid of the beam tail by a filter downstream the gun, for example, using a bend or an $\alpha$ magnet. This method will also be useful for the cold cathode rf gun. For these considerations, the low energy part of beam is excluded in our calculation of the emittance

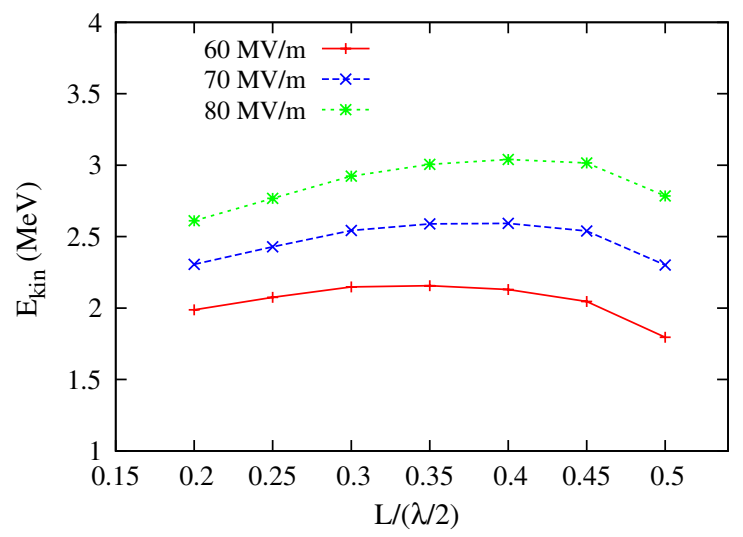

FIG. 7. Beam energy vs cathode cell length.

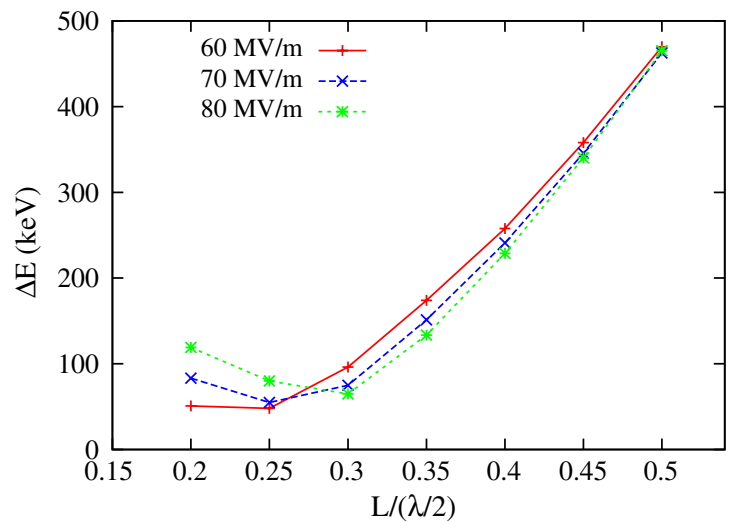

FIG. 8. Energy spread vs cathode cell length.

and rms values and only $85 \%$ of the electrons positioned in the beam head are used in the following discussions.

\section{B. Simulations}

The cathode cell length was varied from 0.20 to 0.50 of $\lambda / 2$. The resonant frequency is tuned to be $2856 \mathrm{MHz}$ and the amplitudes of electric field in the two cells are made the same with SUPERFISH [11]. At the gun exit, the transverse emittance, kinetic energy, and rms energy spread are calculated for the beam head (taking up about $85 \%$ of the total bunch) as plotted in Figs. 6-8.

When the gradient is relatively low $(60 \mathrm{MV} / \mathrm{m})$, the transverse emittance decreases with cathode cell length. With the gradient growing, a minimum appears. The minimum shifts toward a longer cathode cell for higher gradients. For the kinetic energy, a moderate cathode cell is preferred to gain the highest acceleration. If the cathode cell is too long, electrons cannot reach the full cell when the field is strongest. If it is too short, the energy gain in the first cell is small. The energy spread changes in a similar way with the transverse emittance, that is, there is a minimum for a shorter cathode cell. Since the effect of cathode cell length on beam energy is not significant, a shorter cathode cell is preferred to obtain both low emittance and low energy spread beams.

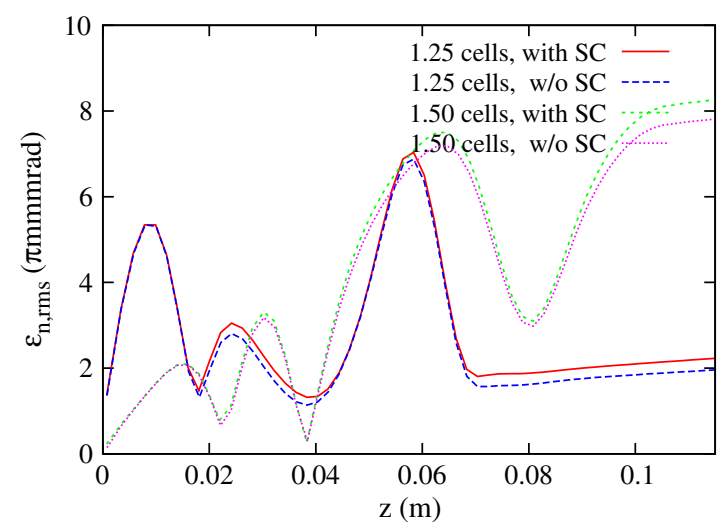

FIG. 9. Transverse emittance along the beam line. 


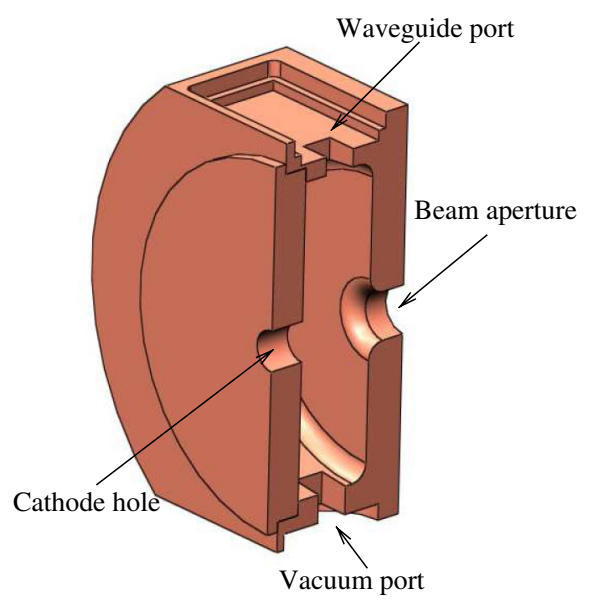

FIG. 10. 3D view of the test cavity.

For the case of $80 \mathrm{MV} / \mathrm{m}$, the evolution of transverse emittance along the beam line for both 1.25 and 1.5 cells gun is shown in Fig. 9. The space charge was turned on and no initial thermal emittance was considered. The transverse emittance for both is hardly affected with the space charge on, unless a much larger amount of charge is involved. While the emittance goes smoothly for the 1.25 cells gun, it rises rapidly for the 1.5 cells gun near the gun exit. That is because the electron bunch reaches the gun exit at a phase away from $\pi / 2$ required by Kim's theory. From simulations, we find that the rf field is changing quickly when the bunch reaches the exit of the 1.5 cells gun. The differing rf focusing forces among slices cause the increase of projected emittance of the bunch.

\section{EXPERIMENTAL RESULTS ON A TEST STAND}

\section{A. The test stand}

A fractional cell has been constructed to study the field emission of electrons from cold cathodes. The 3D model of the cavity and its rf parameters are shown in Fig. 10 and Table I, respectively.

Diamond films are good field electron emitters due to their unique properties such as low threshold field, hardness, and inertness [12]. Our diamond films are fabricated by the microwave plasma chemical vapor deposition method. A base of molybdenum with a radius of $3 \mathrm{~mm}$ is prepared in advance, on which the film is deposited. During the deposition the temperature, pressure and dopant are adjustable to control the quality of the films. The films

TABLE I. Parameters of the test cavity.

\begin{tabular}{lc}
\hline \hline Cavity length $L$ & $2 \mathrm{~cm}$ \\
Resonant frequency $f$ & $2856 \mathrm{MHz}$ \\
Quality factor $Q_{0}$ & 8479 \\
Coupling strength $\beta$ & 1.857 \\
Filling time $\tau$ & $0.33 \mu \mathrm{s}$ \\
\hline \hline
\end{tabular}

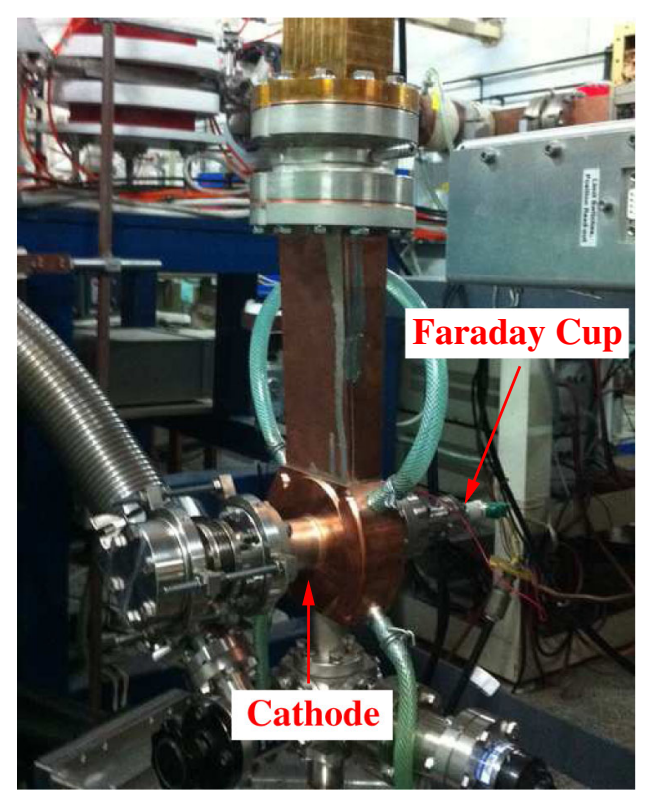

FIG. 11. The test stand.

are then put into the cathode hole of the test cavity and the field-emitted electrons are collected by a Faraday cup outside the cavity. The test stand is shown in Fig. 11.

\section{B. Experimental results and discussions}

Figure 12 showed the measured beam current from a sample as well as the backward wave within a macropulse. The beam current started from about $0.6 \mu \mathrm{s}$. Using the filling time from Table I, it takes roughly $2 \tau$ to build the electric field for observable field emission, as in Fig. 4. The good agreement indicates that the measured beam current indeed came from field emission.

For different samples, we measured the $I-E$ relation as shown in Fig. 13. Here, $I$ and $E$ are the beam current and amplitude of electrical field at steady state as defined in Eqs. (7) and (5), respectively. Dark current was observed from the molybdenum base, which may come from the rough surface necessary for deposition. The highest beam

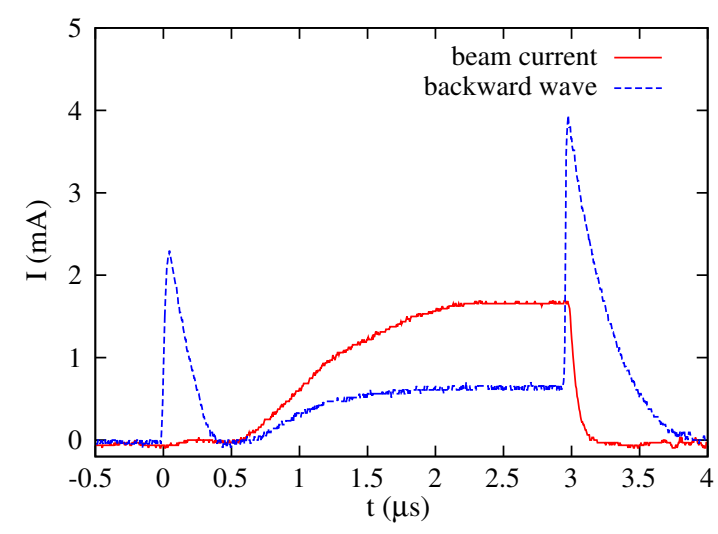

FIG. 12. Beam current and backward wave vs time. 


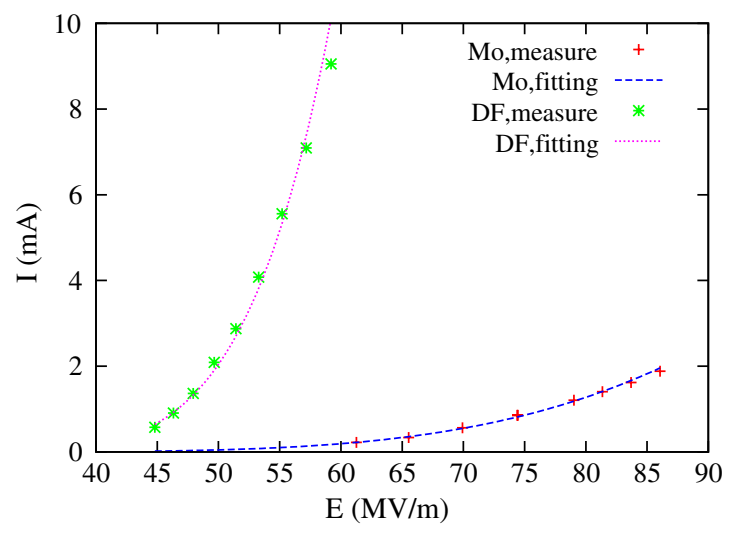

FIG. 13. Beam current for different samples. The diamond films (DF) are deposited on molybdenum (Mo).

current of $9 \mathrm{~mA}$ came from a nitrogen doped diamond film. Such a current corresponds to $3.15 \mathrm{pC}$ in charge or a maximum emission density of $305 \mathrm{~mA} / \mathrm{cm}^{2}$ at the phase of $\pi / 2$. Although the field strength on the cathode surface can reach $90 \mathrm{MV} / \mathrm{m}$, discharge occurred for the DF sample when it exceeded $60 \mathrm{MV} / \mathrm{m}$. The $I-E$ relation was fitted by and agreed well with Eq. (6).

Anyway, the emission from diamond films is still far from our demand of $200 \mathrm{~mA}$. The fabrication condition should be improved so that the films become more emissive and more resistant of discharge.

\section{SUMMARY}

Cold cathode rf guns are thought of as a new type of $\mathrm{rf}$ gun, which has the potential of producing high average current and high quality electron bunches for use in terahertz free electron lasers and others. Field emission is concentrated around the phase of $\pi / 2$ with an rms width of about $15^{\circ}$. For a typical two cells gun, the cathode cell should be shortened for both efficient acceleration and minimization of $\mathrm{rf}$ effect on transverse emittance. A test cavity is built to study the emission of diamond films. We have observed both dark current from the molybdenum base and a beam current of $9 \mathrm{~mA}$ from a nitrogen doped diamond film. Although the extracted beam current is small, the measurement agreed well with theoretical results. In the future, the bunch length and energy spectrum will be measured.

[1] C. Travier, Nucl. Instrum. Methods Phys. Res., Sect. A 304, 285 (1991).

[2] A. Gasper, C. Piel, T. Weis, A. Göhl, T. Habermann, and G. Müller, in Proceedings of the 6th European Particle Accelerator Conference, Stockholm, 1998 (IOP, London, 1998), Vol. 1471.

[3] J. D. Jarvis, B. K. Choi, A. B. Hmelo, B. Ivanov, and C. A. Brau, J. Vac. Sci. Technol. B 30, 042201 (2012).

[4] B. Wang, Y. Xiong, L. Xia, H. Zhang, K. Zhang, and X. Meng, Diam. Relat. Mater. 20, 433 (2011).

[5] R. H. Fowler and L. Nordheim, Proc. R. Soc. A 119, 173 (1928).

[6] F. M. Charbonnier, J. P. Barbour, L. F. Garrett, and W. P. Dyke, Proc. IEEE 51, 991 (1963).

[7] T.P. Wangler, Principles of RF Linear Accelerator (John Wily \& Sons, New York, 1998).

[8] M. Borland, Ph.D. thesis, Stanford University, 1991.

[9] K. J. Kim, Nucl. Instrum. Methods Phys. Res., Sect. A 275, 201 (1989).

[10] K. Flöttmann, http://www.desy.de/ mpyflo/ (2007).

[11] J.H. Billen and L.M. Young, in Proceedings of the Particle Accelerator Conference, Washington, DC, 1993 (IEEE, New York, 1993), pp. 790-792.

[12] J. Robertson, J. Vac. Sci. Technol. B 17, 659 (1999). 